\title{
INTERACTION OF IDEALIZED URBAN INFRASTRUCTURE AND LONG WAVES DURING RUN-UP AND ON-LAND FLOW PROCESS IN COASTAL REGIONS
}

\author{
Nils Goseberg ${ }^{1}$ and Torsten Schlurmann ${ }^{2}$
}

\begin{abstract}
This paper reports experimental results of long wave run-up climbing up a 1:40 sloping beach. The resulting maximum run-up is compared with analytical results and a good agreement is found for single sinusoidal waves with uniform wave period and varying amplitude. Subsequently, the interaction with macro-roughness elements on the beach is investigated for different long-shore obstruction ratios. The reduction in wave run-up is expressed by means of a nomogram relating the wave run-up without macro-roughness elements present to those cases where on-land flow is modified by macro-roughness. The presented results mainly focus on a non-staggered and non-rotated macro-roughness configuration. In addition to the run-up reduction, surface elevation profiles on the shore are presented, that address the shock wave generation when the wave tongue approaches the first row of macro-roughness elements.
\end{abstract}

Keywords: tsunami; long wave; wave run-up; macro-roughness; obstruction ratio

\section{INTRODUCTION AND OBJECTIVE}

\section{Introduction}

In light of the disastrous consequence by major tsunami such as the 2011 Tohoku-Oki tsunami, the understanding of the generation, evolution and run-up of long waves has grown rapidly. To date, the quantification of the maximum run-up of long waves on natural beaches is one of the quintessential questions. In this context it is of primary interest how urbanized coastal areas with macro-roughness (MR) elements (Cox et al. 2009) interact and potentially reduce those hazardous water levels and flow velocities. The severe inundation of the Sendai coast documented by a post-tsunami survey team suitably exemplifies this type of wave-structure interaction where houses and multi-storey buildings withstand the tsunami-induced current until they either collapsed or were washed away (Li et al. 2011). Beside those houses that were damaged there is also evidence that many reinforced houses withstand the severe wave attack.

In the early eighties, experiments addressing the energy loss of a flow interacting with wooden pillars under stationary conditions were published by Goto and Shuto (1983). The authors aimed at investigating the effect of sufficiently solid buildings and low sea walls capable of reducing the overall inundation height numerically and experimentally. They set up experiments where wooden pillars were exposed to varying stationary discharges. The interaction process of an approaching tsunami with urban structures thus is highly transient. In the following an investigation by Ramsden (1993) experimentally and numerically assessed wave forces at vertical walls due to incident long waves represented by dam-break induced bores and surges. It was found that prototype celerities of an approaching tsunami, as deduced from eye-witness reports, agreed reasonably well with accomplished experiments though differences in impact forces were conveyed compared to hydrostatic theory. Further achievements and much more detailed measurements were made either numerically or experimentally. Pedro et al. (2007) investigated the impact of a wave on a square column numerically. Hydrodynamic loads occasioned by impacting waves of a tsunami or a hurricane need to be assessed primarily in order to design constructions appropriately. It was stated that the applied numerical models function sufficiently to obtain design values for constructions, but further insight into hydrodynamical quantities would need more accurate modeling of the situation and thus adequate validation data sets. Important experimental findings were reported by Riggs et al. (2008) who applied either solitary or cnoidal waves resulting in tsunami bores and subsequently measured forces on structural components of a multistory building. Square and rectangular columns in different configurations, horizontal slabs as well as a solid wall were considered while either direct impact or shielded situations were modeled facilitating the demand to enhance existing building codes. For solitary wave impact to an idealized beach front house, Xiao and Huang (2008) deployed a Reynolds-averaged Navier-Stokes solver in a vertical plane. The model, which was validated against two sets of experimental data, was found to reproduce wave run-up reasonably well. Subsequently, forces and overturning moments were analyzed for varying cross-sectional positions of an idealized beach front house.

All the above mentioned studies focused more or less on structural impact in order to improve knowledge on how to cope with loads due to impacting long waves. Yet it is equally important to

\footnotetext{
${ }^{1}$ Franzius-Institute for Hydraulics, Waterways and Coastal Engineering, Leibniz University, Nienburger Str. 4, Hanover, 30167, Germany

${ }^{2}$ Franzius-Institute for Hydraulics, Waterways and Coastal Engineering, Leibniz University, Nienburger Str. 4, Hanover, 30167, Germany
} 
assess how spatially distributed houses affect the overall wave run-up. An analogy could be drawn between the objective of the current work and the effects of riverine flooding, flash flood events or dam breaks that generate hazardous water level rise and resulting inundation. The addressed analogy concerns temporal and spatial evolution of residential area flooding, again involving flow-obstacle interaction. The problem had been numerically tackled by Hashimoto and Park (2008) who expanded the two-dimensional shallow water wave equations besides the well-known bottom roughness terms accounting for bottom shear stress with an additional term accounting for drag forces induced by the presence of buildings. The model which was validated against a flood disaster in Fukuoka, Japan explicitly differentiates four obstacle types attributed with drag coefficients and also accounts for building density. Lately Cox et al. (2009) scrutinized a portion of the town Seaside, Oregon with a hydraulic model of $1: 50$ length scale. Though partially idealized, many of the existing features such as the seawall and a major hotel were kept realistic in order to investigate the propagation of an approaching bore front developed out of an error function-induced or solitary wave. The flow properties were recorded by means of conventional sensors as well as by an adapted video system to provide high-resolution space-time information of the wave propagations through urban macro-roughness. Finally, experimental results were compared to calculations accomplished by two individual numerical tools and good agreement was obtained. Those experiments were particularly helpful to assess the tsunami hazard at Seaside.

Apart from this achievements, another research question that is still unacknowledged is how to generalize buildings in the proximity of the shoreline under the attack of a tsunami wave and how to parametrize the energy loss of transient flow reqimes on the shore. It is further still unknown how the overall wave run-up is influenced for varying obstruction ratios.

\section{Objective}

The objective of this paper is thus to investigate experimentally how tsunami-like long waves running up a plain, sloping beach behave and how the approaching wave interacts with MR-elements representing urban settlements directly at the shore. This near- and onshore interaction results in reduced wave run-up that has to be quantified depending on the most important parameters such as the long-shore obstruction ratio. In order to achieve this goal for very long waves in limited laboratory space it was necessary to find an appropriate wave generation mechanism capable of generating arbitrary long waves.

\section{EXPERIMENTAL SETUP}

The experiments were carried out in a closed-circuit wave flume at the Franzius-Institute for Hydraulic, Waterways and Coastal Engineering depicted in Fig. 1. For the purpose of generating tsunami-like long waves the closed-circuit flume was divided into a propagation and a reservoir section by a separation wall.

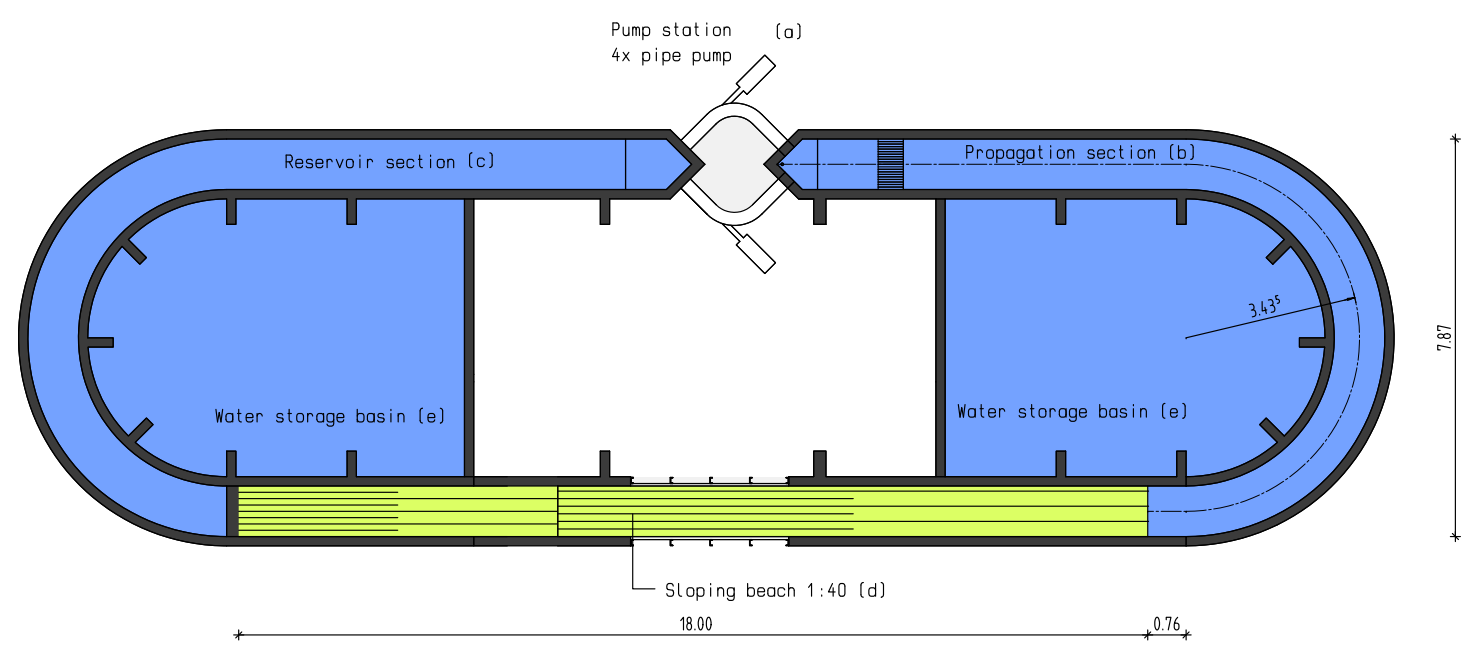

Figure 1. Schematic drawing of the closed-circuit flume and its equipments, (a) pump station, (b) propagation section, (c) reservoir section, (d) sloping beach, (e) water storage basin

The pump station holding a maximum pump capacity of $500 \mathrm{~m}^{3} / \mathrm{h}$ indicated as position (a) in Fig. 1 drives the water in the flume in either forward or backward direction. Water pumped into forward 
direction propagates along the propagation section marked as position (b). The generation of long waves is accomplished by means of this set of high-capacity pipe pumps which is under the control of a proportional-integral-derivative controller. Water that is pumped out of the propagation section is stored in the reservoir section (c) until the experimental process requires an additional volume of water. The sloping beach section denoted as position (d) models the bathymetry of a natural beach and its adjacent coastal hinterland.

An beach wedge with 1:40 slope was installed in the straight section of the flume as a fixed bed setup. The still water depth is kept constant from the pump station to the toe of the ramp amounting to $d=0.3 \mathrm{~m}$. The experiments are scaled with Froude similitude at a length scale of $1: 100$. The toe of the beach is situated $0.76 \mathrm{~m}$ away from the end of the 180 curve. The surface of the artificial ramp is constructed of unplasticized polyvinyl chloride (PVC). Fig. 2 illustrates the idealized beach situation that was modeled in cross-section and top view.

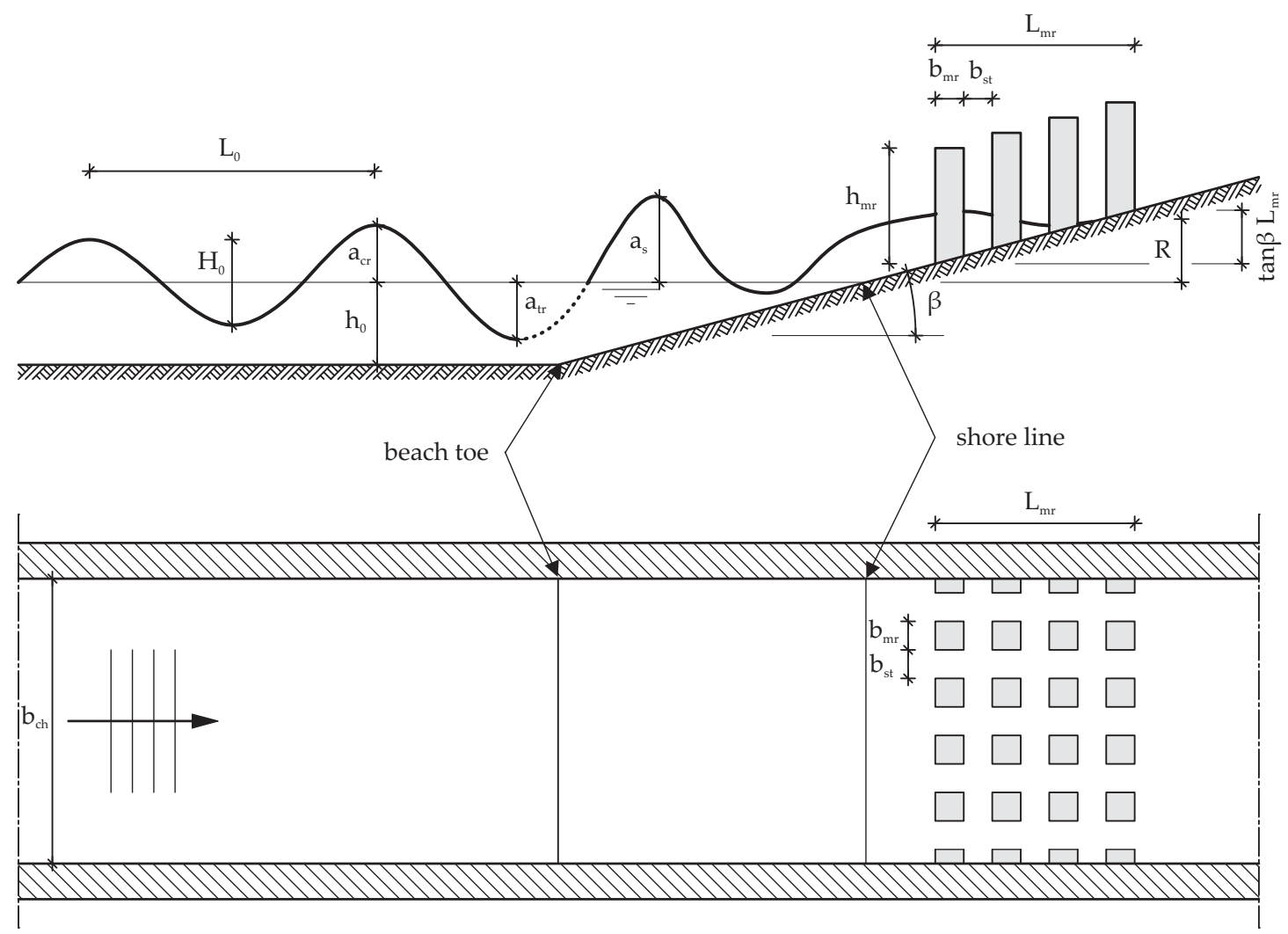

Figure 2. Schematic sketch of the modeled beach situation attached to a region of uniform water depth, MR-elements are present on the shore, upper panel depicts a cross-section, lower panel gives top view

The pump station (Fig. 3) which accelerates or decelerates the volume of water is located opposite to the beach slope. The pumps are controlled by an elementary feedback control system which generally includes three components, a plant (i.e. an actuator, pump valve), a sensor which is meant to measure the output of the plant under control and the controller itself which assures the input of the plant (Ang et al. 2005).

The actual variable value is measured by a pressure sensor situated directly at the output of the pumps. The controller performance is optimally tuned when the controller reduces the error between the predefined input signal (set point) to the plant and the measured sensor input (process or control variable) most. For the results described in this paper the controller has been tuned on a trial-and-error basis. A more detailed discussion on the flume outline, its functionality for the long wave generation and the features of the pump-driven wave maker are given in Goseberg and Schlurmann (2010), Goseberg (2011), Goseberg (2012).

The run-up onto the beach wedge with 1:40 slope is investigated for a single, sinusoidal wave form with $T=60$ s while the wave height has been increased stepwise from $50 \%-100 \%$. An amplitude of 


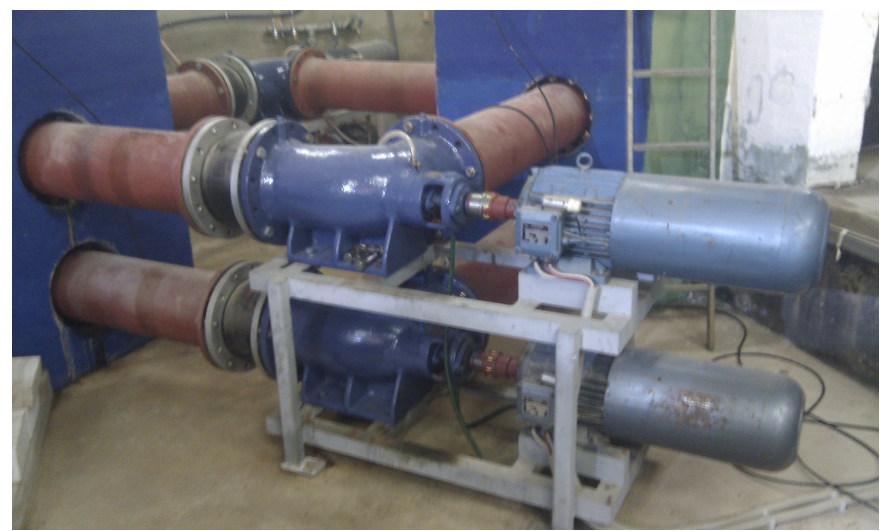

Figure 3. Image of the pump station controlled by the PID-controller, during the experiments only the lower two pumps were used due to water depth constraints

$100 \%$ corresponds $0.04 \mathrm{~m}$ in model scale. The tested single sinusoidal waves were always leading depression waves. Run-up experiments and those where the approaching long wave interacts with MR-elements on the beach have been repeated at least twice in order to assure correct results. Few wave MR-element combinations were also repeated more often in order to investigate how repeateable the experiments were. From those experimental runs showing consistent results it was decided that two repetitions per combination are sufficient.

Fig. 4 depicts four different MR-element combinations which principally affect the long wave run-up. Further combinations are additionally feasible though in this paper the focus is only placed on the aligned, non-rotated combination of MR-elements. MR-elements were fabricated as cube-shaped blocks from concrete with an edge length of $10 \mathrm{~cm}$. Besides the general placement of the MR-elements distances in long- and cross-shore direction were varied. Additionally, the number of MR-element rows parallel to the shoreline was also varied $(1,5,10$ rows).
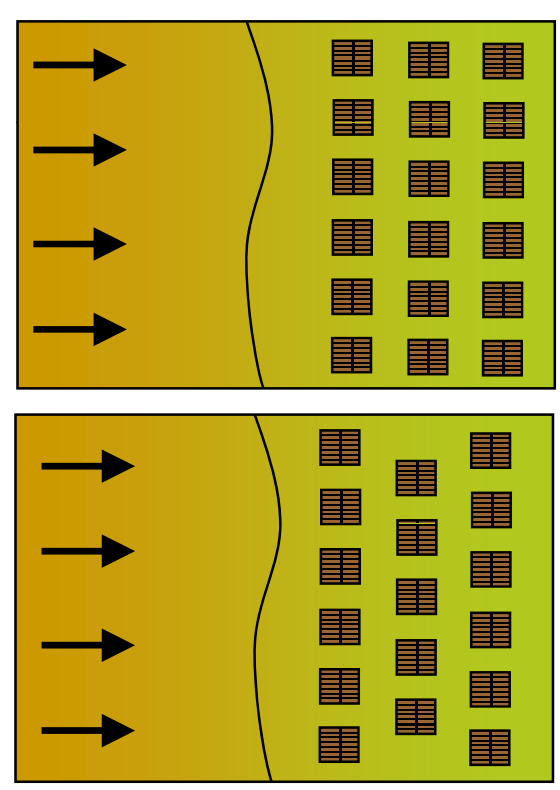
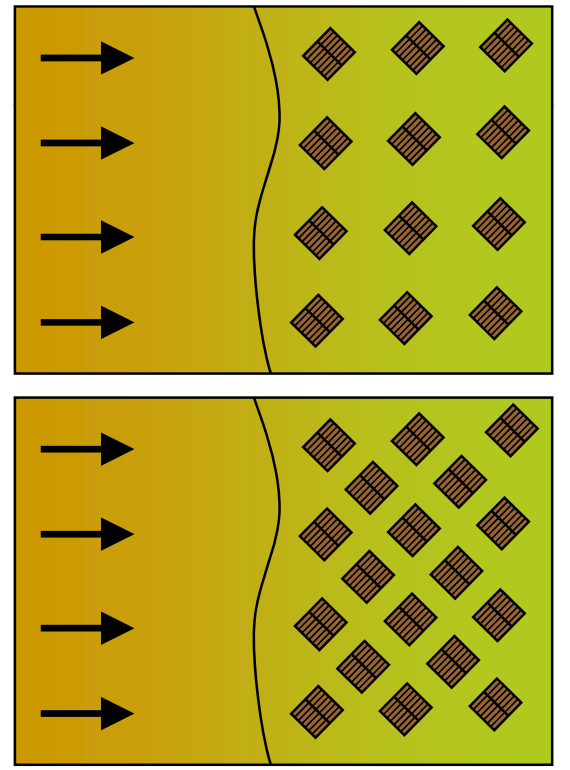

Figure 4. Combinations of MR-element arrangement that could affect the long wave run-up, upper left panel: aligned and non-rotated, upper right panel: aligned and rotated, lower left panel: staggered and non-rotated, lower right panel: staggered and rotated 


\section{WAVE MAKER CAPABILITIES}

In order to present how the wave maker performs a comparison of a possible tsunami water surface time series and the generated instantaneous water level at the wave maker position is chosen. Since no in-situ measurements of actual tsunami time series were at hand, a resulting numerically derived time series for the city of Pandang, Indonesia was applied to illustrate the capability of the wave maker to generate arbitrary long waves. For details of the numerical modeling the reader is referred to (Goseberg and Schlurmann 2009) and (Schlurmann et al. 2010). According to the chosen experimental length scale of 1 : 100 the time series was extracted from the numerical results at a water depth of $d=30 \mathrm{~m}$ offshore of the Padang coastline and slightly adjusted in terms of the wave period.
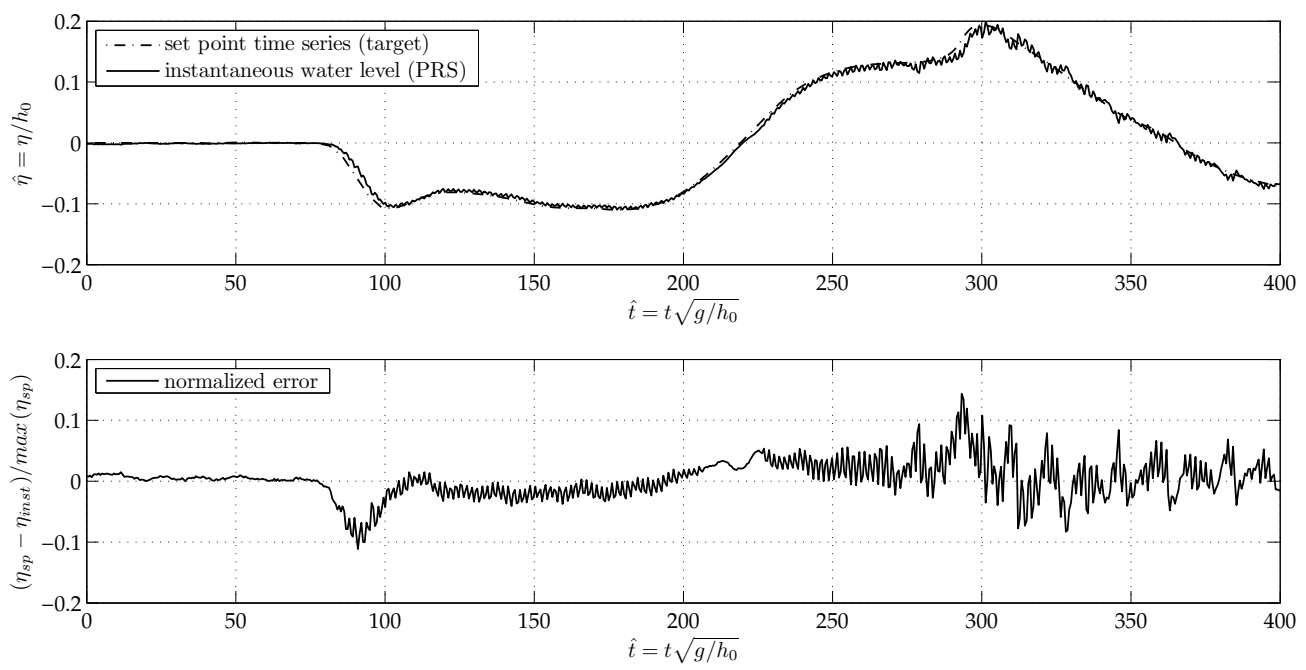

Figure 5. Illustration of the wave maker performance for a prototype waveform deduced from numerical modeling for the city of Padang with $T=50.0 \mathrm{~s}, h_{0}=0.3 \mathrm{~m}, a_{t r}=0.03 \mathrm{~m}$ and $a_{c r}=0.06 \mathrm{~m}$, upper sub-figure of the figure depicts the non-dimensional water surface elevation $\eta / h_{0}$ of the set point signal and the control variable in regard to the dimensionless time $\hat{t}=t \sqrt{g / h_{0}}$, lower sub-figure shows the control error normalized by the amplitude $a_{c r}$ of the set point signal over non-dimensional time

After eventually adjusting the PID-controller sufficiently it can be seen, that the errors are small and a step-up of oscillations that would normally appear without proper PID-tuning is successfully prevented with the controller setup utilized. Minor deviations arise at the beginning of the monotonically non-increasing wave trough $(\hat{\eta}=80-100)$. The control error, which amounts to -0.1 as a peak, rather stems from the control variable lagging behind the set point curve. The oscillations, especially in the downward movement of the water surface, remain small and the controller action results in a time lag. The following plateau phase is characterized by minor oscillations. A positive control error arises as soon as the wave crest has to be generated by the wave maker. During all of the experiments it had always been more problematic to generate wave crests than wave troughs. Besides the described control errors the overall appearance of the generated long, tsunami-like wave is sufficient to proceed with run-up experiments. For details regarding the propagation along the flume axis, the reader is again referred to (Goseberg 2011).

\section{RESULTS}

Run-up

The experimental run-up results are going to be presented in the following. The objective of the section is to elucidate the run-up of long waves on a plane, 1:40 sloping, impermeable beach. No macro-roughness elements are considered on the beach firstly in order to evaluate the wave run-up without disturbing effects. The outcome of the analysis is then compared to existing theoretical approaches and to experimental results found in literature.

In the present study the wave period $T$ and the beach slope $\beta$ were not varied. Yet the wave height was varied instead in order to ease the experimental procedure. The resulting wave run-up normalized by the crest amplitude can be related to the surf similarity parameter $\xi$. Madsen and Schäffer (2010) 
published an analytical derivation of run-up formula for various wave forms basing on convolution integrals. Wave forms such as isoceles $\mathrm{N}$-waves, single waves and sinusoidal waves are covered but with the important exception that these waveforms did no longer exhibit a tie to wave height-to-depth ratio. On the basis of assuming linear waves at the beach toe Madsen and Schäffer (2010) proposed the following expressions for the amplification ratio of incoming waves and the associated velocities

$$
\frac{R_{\text {up } / \text { down }}}{A_{0}}=\chi_{\text {elev }}\left(\frac{\Omega^{2} h_{0}}{g \gamma^{2}}\right)^{1 / 4}, \quad \frac{\gamma V_{\text {up/down }}}{\Omega A_{0}}=\chi_{\text {velo }}\left(\frac{\Omega^{2} h_{0}}{g \gamma^{2}}\right)^{1 / 4},
$$

where $R=$ run-up/run-down, $A_{0}=$ offshore wave amplitude, $\chi_{\text {elev }}$ and $\chi_{\text {velo }}=$ shoreline elevation and velocity, $\Omega=2 \pi / T=$ a duration in analogy to the common wave period, $h_{0}=$ offshore water depth, $g=$ acceleration due to gravity and $\gamma=$ beach slope. The normalized run-up/run-down according to their theory is summarized in the Eq. (2) as a function of the surf similarity parameter

$$
\frac{R_{\text {up } / \text { down }}}{A_{0}}=\chi_{\text {elev }} \pi^{1 / 4}\left(\frac{A_{0}}{h_{0}}\right)^{-1 / 4} \xi^{-1 / 2}
$$

It is thus possible to compare experimental run-up results to the analytical expression (Eq. (2)). As shown in Fig. 6 the maximum relative run-up, which is normalized by the positive amplitude at the wave maker, decreases with an increasing surf similarity parameter $\xi_{\infty}$. The increase in non-linearity, which can be expressed as the ratio of the applied wave amplitude of the crest $a_{c r} / h_{0}$, reduces the surf similarity of the applied wave condition at the beach under investigation. Higher non-linearity of the wave also indicates higher energy content. This finding underlines the statement of Madsen and Fuhrman (2008) where critical flow depth and flow velocities occur for surf similarity in the order of $\xi_{\infty}=3-6$. As can be seen in Fig. 6 experimental results compare reasonably well with analytical predictions supporting the underlying assumptions. The experimentally derived surf similarity was calculated using the wave properties measured at the beginning of the propagation section.

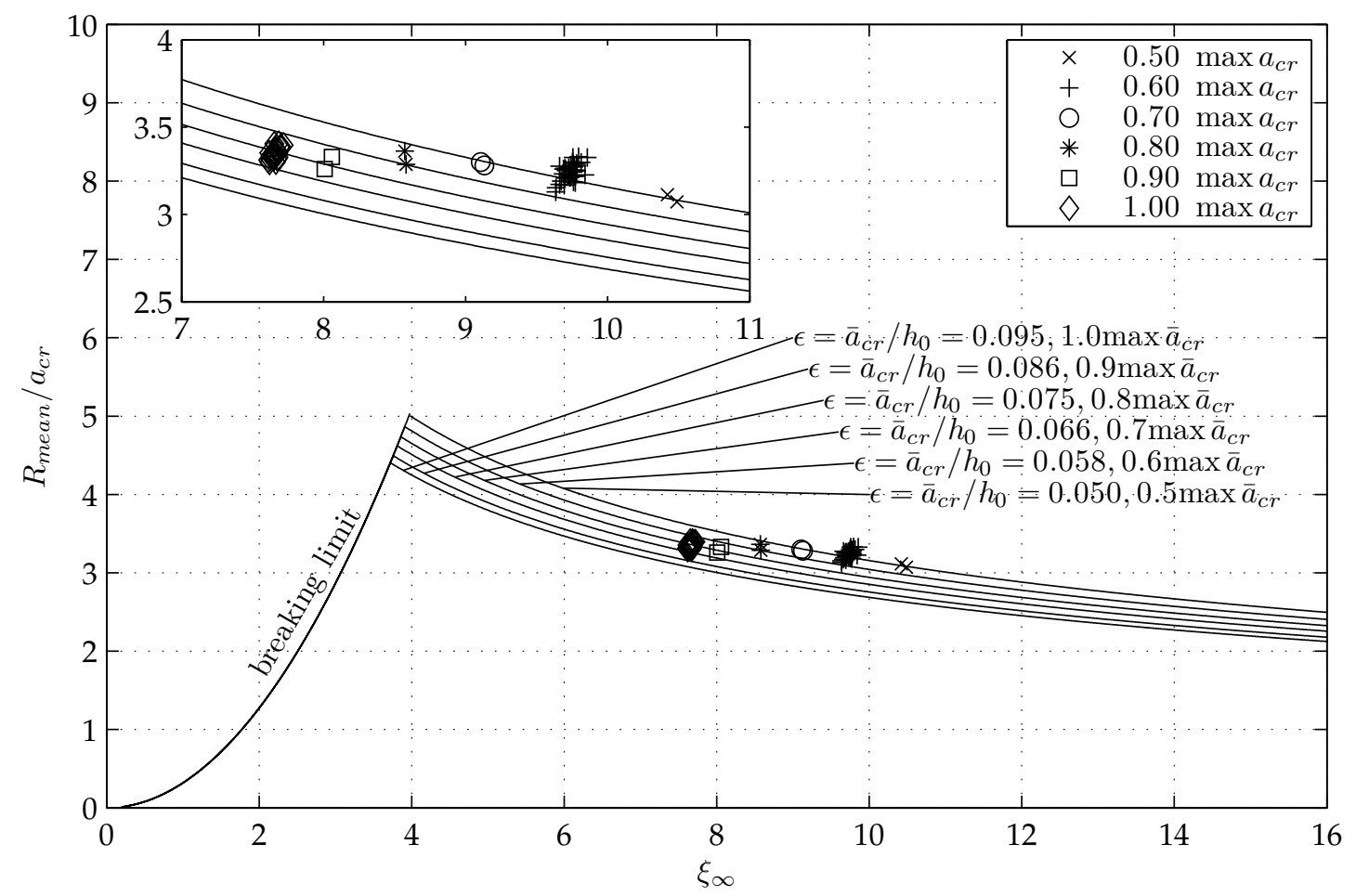

Figure 6. Maximum relative run-up as a function of the surf similarity parameter $\xi$ depicted by the markers and according to the applied waves, the theoretical breaking limit given by Eq. (5.3.2) and the theoretical run-up functions given by Eq. (2). The inset zooms to the experimental data.

Yet it is apparent that the results do not perfectly fit the expected analytical behavior even though the results already indicate that the theory and the functional relation of the non-dimensional terms is 
well established. One reason for this could be that the analytical approach fully neglects the influence of surface roughness while the experimental setup aims at eliminating roughness influences to the greatest extent within the limitations of model and scale effects.

\section{Water Surface Elevation}

The qualitative influence of the macro-roughness element configuration to the spatial distribution of the water level at distinct time steps should be assessed in the following. For this purpose a staggered, rotated MR-element configuration is chosen that reduces the wave run-up most. The number of MR-element rows is set to $n=5$ for the presented results. The smallest street width $b_{s t}$ is utilized for the plots presented in Fig. 7 in order to highlight the qualitative effects of the MR-elements. The position and the height of the obstacle area is given by the dash-dotted gray lines while the beach surface is presented by the full gray line. A wave with a non-linearity of $\epsilon=0.093$ is used. Out of the sequence of available time steps of the wave evolution along the beach wedge two specific times are presented in order to focus on the basic features. The reported times refer to the beginning of the wave generation and it thus has to be accounted for the time needed until the wave has propagated along the region of uniform water depth.
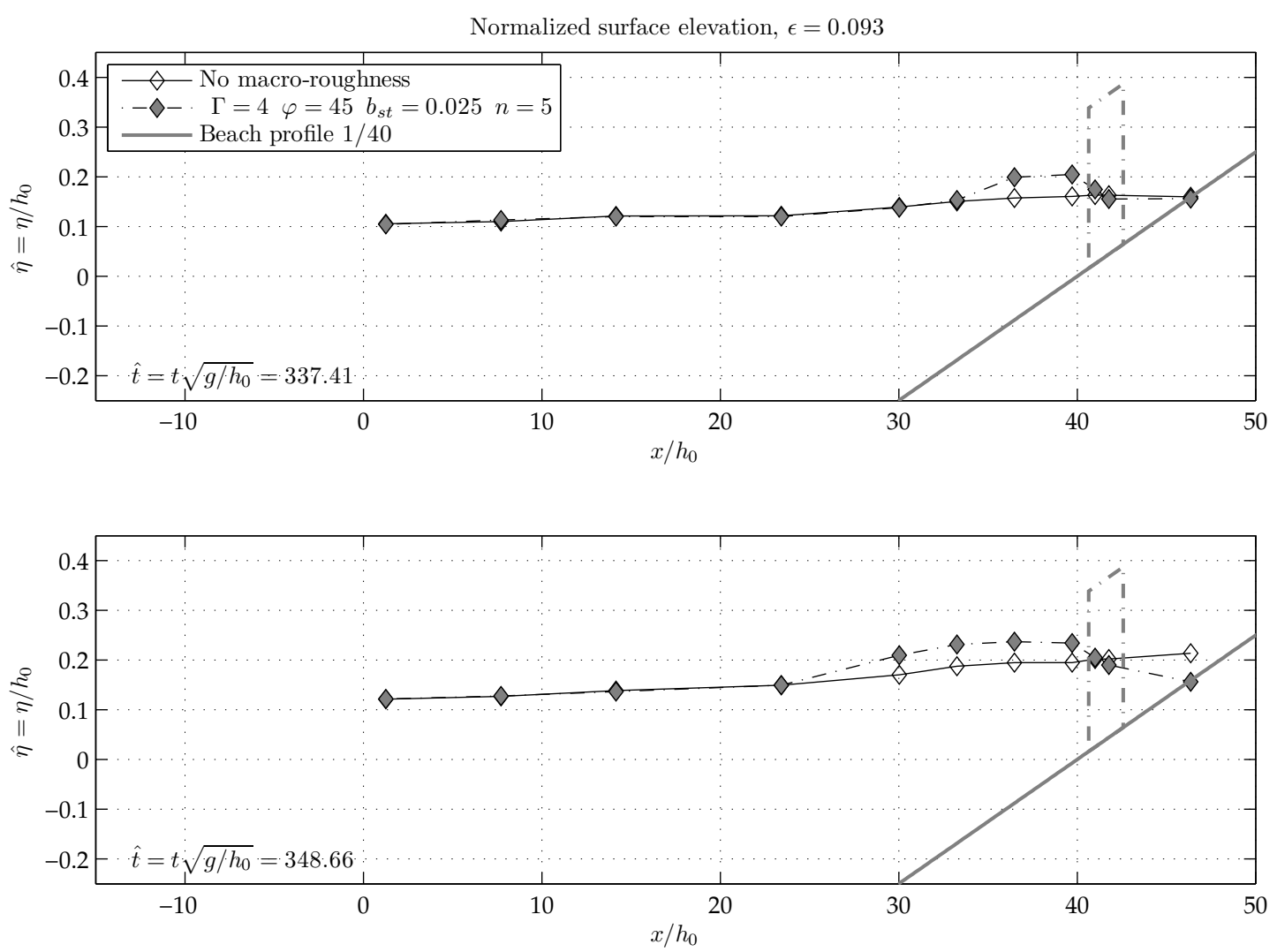

Figure 7. Comparison of the normalized surface elevation for a single, sinusoidal wave with $\epsilon=0.093$ at $\hat{t}=494.92$ and $\hat{t}=517.42$ with and without the presence of macro-roughness elements. The MR-element combination is staggered with rotated obstacles, $b_{s t}=0.025$ and $n=5$. Surface elevation and spatial coordinates are normalized by the invariant water depth $h_{0}=0.3 \mathrm{~m}$. The dash-dotted line depicts the beginning of the MR-element area.

The approaching wave tongue collides with the first macro-roughness element row and a shock wave is formed which propagates seawards. This feature is observable for all investigated street widths and for all different MR-element combinations. The height of the shock wave however is small due to the fact that lesser obstruction ratios and thus smaller reflective vertical surfaces are involved in this rotated case. The front of the initiated shock wave is comparable with a monoclinal rising wave which in contrast to the definition is rapidly varying during its seaward propagation. In the present case the surface gradient of the positive surge, whose front is initially steep, decreases rapidly and it flattens out noticeably. On 
the other hand it is noticeable that wave propagation onto the shore becomes delayed when MR-elements interact with the approaching wave.

\section{Macro-roughness influence}

The following section is finally going to analyze the given objective of the interaction of long, tsunami-like waves with the proposed macro-roughness element configurations and the alleged reduction of the relative run-up due to the obstruction imposed by the macro-roughness elements. The interaction process leading to a final reduction of wave height depends amongst others on the long- and cross-shore obstruction ratio as well as the way how MR-elements are arranged on the beach. Goseberg (2011) found out that run-up reduction is mostly influenced by increasing long-shore obstruction ratio. Hence, in this paper it is shown how the run-up is reduced depending on this ratio for the aforementioned aligned, non-rotated MR-element combination. The long- and cross-shore obstruction ratios $\psi_{c s}$ and $\psi_{l s}$ are defined as follows:

$$
\begin{gathered}
\psi_{l s}=\frac{\hat{b}_{m r}}{\hat{b}_{m r}+b_{s t}}=\frac{b_{m r} / \cos \varphi}{b_{m r} / \cos \varphi+b_{s t}} \\
\psi_{c s}=\frac{L_{m r}}{n \hat{b}_{m r}}=\frac{n\left(\hat{b}_{m r}+b_{s t}\right)-b_{s t}}{n \hat{b}_{m r}}=\frac{n\left(b_{m r} / \cos \varphi+b_{s t}\right)-b_{s t}}{n b_{m r} / \cos \varphi}
\end{gathered}
$$

where $\hat{b}_{m r}=b_{m r} / \cos \varphi$ denotes the width of the macro-roughness elements according to their angle of rotation and with respect to the incident wave direction. The long-shore rate of obstruction increases according to Eq. (3) when the ratio of the macro-roughness width to the sum of the street width plus the macro-roughness element width tends to unity. A long-shore rate of obstruction of unity is equivalent to a complete obstruction of the beach and results in full reflection of the wave and the run-up tongue. The cross-shore rate of obstruction depends on the ratio of the number of element rows times the width to the length of the macro-roughness area in on-shore direction. This geometrical ratio accounts for the obstruction exposed to the in-land propagating flow. The ratio $\psi_{c s}$ decreases if the street width tends to zero, $b_{s t} \rightarrow 0$. The ratio grows if the number of macro-roughness rows parallel to the shoreline increases. The length of the obstructed area in the direction of wave propagation is given by Eq. (5):

$$
L_{m r}=n\left(b_{m r}+b_{s t}\right)-b_{s t}
$$

From an engineering perspective it is preferable to either yield empirical equations or nomograms which are suitable to predict the run-up reduction due to a given macro-roughness combination in comparison with an undisturbed cases. Fig. 8 illustrates the relation of the relative run-up $R_{m e a n} / a_{c r}$ to the surf similarity parameter $\xi_{\infty}$.

The reduced relative run-up according to the long-shore obstruction ratio is plotted as an error bar line where the line denotes the averaged run-up of the number of MR-element rows parallel to the shoreline ( $n=1, n=5$ and $n=10$ ). The error bars mark the standard deviation to the plotted average values. Generally a smaller relative run-up is associated with a higher number of macro-roughness element rows. The relative run-up associated with the long-shore obstruction ratio decreases slightly with an increasing non-linearity of the applied waves, which is contributed to greater momentum exchange within the macro-roughness element area. The six error bars for each cross-shore obstruction ratio always assign the six applied wave conditions. The labels which indicate the non-linearity of the waves refer to all error bars arranged in the vertical. Generally it is obvious that the highest long-shore obstruction ratio decreases the relative run-up most.

\section{SUMMARY AND CONCLUSIONS}

The objective of this study was the analysis of the interaction between the long wave run-up on a plane beach representing tsunami attack and coastal urban structures. Long sinusoidal waves of a single period were utilized to study the wave motion. The interaction of macro-roughness elements, mimicking coastal urban settlements, with these waves is the focal point of the study. Additionally, the behavior of the long waves was also investigated when climbing up a plane beach fully undisturbed. A novel pump-driven wave generation methodology is firstly proposed. The generation of arbitrary long waves is achieved by means of pipe pumps which accelerate or decelerate a volume of water in horizontal direction under the control of a feedback loop system. It accounts for the fact that tsunami are basically shallow water waves where the particle velocity is homogeneously distributed over the water 


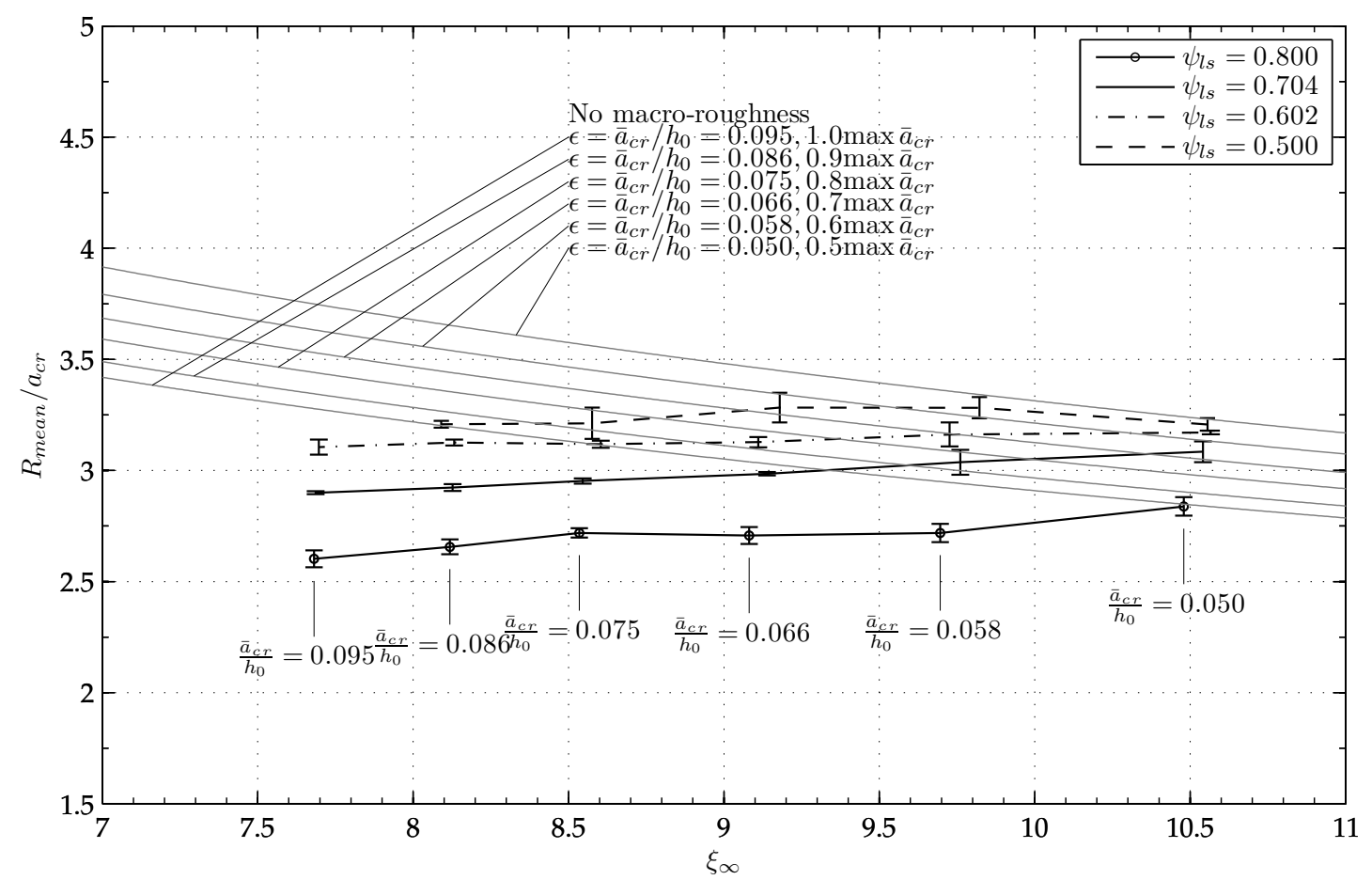

Figure 8. The relative wave run-up $R_{\text {mean }} / a_{c r}$ as a function of the surf similarity parameter $\xi_{\infty}$ for an aligned and non-rotated MR-element combination. The experimental data are plotted according to the individual cross-shore obstruction ratio. The error bars indicate the variation of the number of macro-roughness element rows. The gray parallel graphs indicate the relative run-up without the interaction with macro-roughness according to the analytical results (cp. previous section). The non-linearity of the applied waves is indicated as text which always refers to all error bars in the vertical above.

depth. The experimental reproduction of numerically deduced surface elevation time series proved the applicability of the novel wave generation technique. The experimentally determined relative run-up of single, sinusoidal waves on a plane beach without macro-roughness elements agrees well with an analytical approach by (Madsen and Schäffer 2010). The relative run-up of long sinusoidal waves is significantly influenced by the presence of macro-roughness elements onshore. A nomogram is given to allow for the determination of the relative run-up reduction for a aligned, non-rotated MR-element combination, various distances between the single obstacles and a range of wave non-linearities. Simple yet idealized predictions of the reduction of long waves due to macro-roughness element configurations are therewith made possible.

\section{ACKNOWLEDGMENTS}

This work was partly funded by the 'TsunGen'-project through internal funds of Gottfried Wilhelm Leibniz University Hanover, Germany. The authors also acknowledge the partial support through the DFG/BMBF special Programme Geotechnologies - Early Warning Systems in Earth Management, Sponsorship Code: 03G06666B.

\section{REFERENCES}

Ang, K., G. Chong, and Y. Li. 2005. PID control system analysis, design, and technology, IEEE Transactions on Control Systems Technology, 13(4), 559-576.

Cox, D., T. Tomita, P. Lynett, and R. Holman. 2009. Tsunami inundation with macro-roughness in the constructed environment, J. M. Smith (Ed.), Proceedings of the International Conference on Coastal engineering, Volume 2, 1421-1432. World Scientific.

Goseberg, N.. 2011. The Run-up of Long Wave - Laboratory-scaled Geophysical Reproduction and Onshore Interaction with Macro-Roughness Elements. Ph. D. thesis, Leibniz University Hannover, Hannover, Germany. 
Goseberg, N.. 2012, June. A laboratory perspective of long wave generation, Proceedings of the Twenty-second (2012) International Offshore and Polar Engineering Conference, Volume 3, Rhodes, Greece, International Society of Offshore and Polar Engineers (ISOPE), 54-60.

Goseberg, N. and T. Schlurmann. 2009, July. Enhanced hazard mapping on a medium-resolved numerical grid for the city of padang, west sumatra, Journal of Ship Technology, 5(2), 13-21.

Goseberg, N. and T. Schlurmann. 2010. Numerical and experimental study on tsunami run-up and inundation influenced by macro roughness elements, P. Lynett and J. M. Smith (Eds.), Proceedings of the International Conference on Coastal Engineering.

Goto, C. and N. Shuto. 1983. Effects of large obstacles on tsunami inundations, K. Iida and T. Iwasaki (Eds.), Tsunamis: Their Science and Engineering, Chapter Tsunami Run-up, 551-525. Tokyo/Reidel, Dordrecht, Terra Science Pub. Co.

Hashimoto, H. and K. Park. 2008. Flood Recovery, Innovation and Response I, Chapter Two-dimensional urban flood simulation: Fukuoka flood disaster in 1999, 369-384. WIT press.

Li, R., S. Liu, Q. Guan, and Y. Peng. 2011. Post-disaster assessment of northeastern coastal region for the 2011 sendai earthquake and tsunami, ISWREP 2011 - Proceedings of 2011 International Symposium on Water Resource and Environmental Protection, 3, 2429-2432.

Madsen, P. A. and D. R. Fuhrman. 2008. Run-up of tsunamis and long waves in terms of surf-similarity, Coastal Engineering, 55(3), 209 - 223.

Madsen, P. A. and H. A. Schäffer. 2010. Analytical solutions for tsunami runup on a plane beach: single waves, n-waves and transient waves, Journal of Fluid Mechanics, 645, 27-57.

Pedro, H., K.-W. Leung, M. Kobayashi, and H. Riggs. 2007. Numerical study of the wave impact on a square column using large eddy simulation, Proceedings of the International Conference on Offshore Mechanics and Arctic Engineering - OMAE, Volume 3, 1015-1021.

Ramsden, J. D.. 1993. Tsunamis: Forces On A Vertical Wall Caused By Long Waves, Bores, And Surges On A Dry Bed. Ph. D. thesis, California Institute of Technology, Pasadena, California.

Riggs, H., I. N. Robertson, K. F. Cheung, G. Pawlak, Y. L. Young, and S. C. Yim. 2008. Experimental simulation of tsunami hazards to buildings and bridges, Proceedings of 2008 NSF Engineering Research and Innovation Conference, Knoxville, Tennessee.

Schlurmann, T., W. Kongko, N. Goseberg, D. H. Natawidjaja, and K. Sieh. 2010. Near-field tsunami hazard map padang, west sumatra: utilizing high resolution geospatial data and reseasonable source scenarios, P. Lynett and J. M. Smith (Eds.), Proceedings of the International Conference on Coastal Engineering.

Xiao, H. and W. Huang. 2008. Numerical modeling of wave runup and forces on an idealized beachfront house, Ocean Engineering, 35(1), 106-116. 